\title{
Ergodicity for Singular-Degenerate Stochastic Porous Media Equations
}

\author{
Marius Neu $\beta^{1}$
}

Received: 29 May 2020 / Revised: 14 December 2020 / Accepted: 1 February 2021

(c) The Author(s) 2021

\begin{abstract}
The long time behaviour of solutions to generalized stochastic porous media equations on bounded intervals with zero Dirichlet boundary conditions is studied. We focus on a degenerate form of nonlinearity arising in self-organized criticality. Based on the so-called lower bound technique, the existence and uniqueness of an invariant measure is proved.
\end{abstract}

Keywords Generalized stochastic porous media · Invariant measures · Ergodicity · Self-organized criticality

Mathematics Subject Classification 37A25 · 76S99 · 35K59 • 60H15

\section{Introduction}

We consider the singular-degenerate generalized stochastic porous media equation

$$
\begin{aligned}
\mathrm{d} X_{t} & \in \Delta\left(\phi\left(X_{t}\right)\right) \mathrm{d} t+B \mathrm{~d} W_{t}, \\
X_{0} & =x_{0},
\end{aligned}
$$

on a bounded interval $\mathcal{O} \subseteq \mathbb{R}$ with zero Dirichlet boundary conditions. The multi-valued function $\phi$ is the maximal monotone extension of

$$
\mathbb{R} \ni x \mapsto x \mathbf{1}_{\{|x|>1\}},
$$

$W$ is a cylindrical Wiener process on some separable Hilbert space $U$, and the diffusion coefficient $B$ is an $L^{2}(\mathcal{O})$-valued Hilbert-Schmidt operator satisfying a non-degeneracy condition (see (2.5) below). Equation (1.1) is understood as an evolution equation on $H^{-1}$, the dual of $H_{0}^{1}(\mathcal{O})$, where it can be solved uniquely in the sense of SVI solutions, as shown in [40]. The main result of the present work is the existence and uniqueness of an invariant probability measure for solutions to (1.1).

\footnotetext{
$凶$ Marius Neuß

neuss@mis.mpg.de

1 Max-Planck-Institut für Mathematik in den Naturwissenschaften, 04103 Leipzig, Germany
} 
The above form of stochastic porous media equations is motivated by the analysis of non-equilibrium systems, appearing in the context of self-organized criticality (for a survey, see e.g. [48]). Self-organized criticality is a statistical property of systems displaying intermittent events, such as earthquakes, which are activated when the underlying system locally exceeds a threshold. These dynamics are reflected by the discontinuity and degeneracy of the nonlinearity $\phi$ above. In order to get a better understanding of the long-time behaviour of these systems, we prove the existence of a unique non-equilibrium statistical invariant state for (1.1). Since this is the candidate to which the transition probabilities are expected to converge for long times, it is the key object for the statistical behaviour of the respective process.

A previous approach to the long-time behaviour of Markov processes stemming from monotone SPDEs with singular drift, by which the present article is inspired, is [32], which in turn uses the more abstract framework of [31]. In these works, the existence and uniqueness of invariant probability measures to stochastic local and non-local $p$-Laplace equations is proved, where the multivalued regime $p=1$ is included. In one dimension, the paradigmatic case is the equation

$$
\mathrm{d} X_{t}=\Delta\left(\operatorname{sgn}\left(X_{t}\right)\right)+\mathrm{d} W_{t},
$$

where sgn denotes the maximal monotone extension of the classical sign function. The proof relies on sufficient criteria from [35], where the so-called lower bound technique has been extended to Polish spaces which are not necessarily locally compact. This technique relies on the existence of a state being an accessible point for the time averages of the transition probabilities uniformly in time, and the so-called "e-property", which is a uniform continuity assumption on the Markov semigroup. To verify these criteria, the focus of [32] rests on energy estimates to first bound the mass of these averages to $L^{m}$ balls for some suitably chosen $m \in(2,3]$. As a next step, the convergence to a chosen accessible state with probability bounded below is shown, which is done by comparing the solution of (1.3) to a control process, which obeys the mere deterministic dynamics of (1.3), i.e.

$$
\begin{aligned}
\frac{\mathrm{d}}{\mathrm{d} t} X_{t} & =\Delta\left(\operatorname{sgn}\left(X_{t}\right)\right), \\
X_{0} & =y,
\end{aligned}
$$

for $y \in L^{m},\|y\|_{m} \leq R$ for some $R>0$. In this, simpler setting than (1.1), there is a unique limiting state to (1.4) which is a natural candidate for the aforementioned accessible point.

In the present article, we aim to prove the existence and uniqueness of an invariant probability measure by similar ideas. While energy estimates for (1.1) are easier to obtain due to the linear growth of $\phi$ (cf. (1.2)) at $\pm \infty$, the degenerate form of the nonlinearity destroys the convergence of the noise-free system to a unique fixed point. This is why we have to add a forcing term to the control process and rely on a more refined deterministic analysis of the resulting inhomogeneous monotone evolution equation. To guarantee the convergence of this modified control process, the forcing term has to be sufficiently non-degenerate, and as the connection of the solution to (1.1) to the control process only works if the noise is "close" to the deterministic forcing with non-zero probability, this relies on some non-degeneracy requirements on the noise. As in [32], it is important that the convergence of the deterministic process takes place uniformly for initial values in sets of bounded energy. We tackle this problem with the help of a comparison principle, which, however, only works if the energy actually controls the $L^{\infty}$ norm. This leads to the restriction to one spatial dimension. Finally, most of the above-mentioned steps have to be argued on an approximate level due to the 
singularity of the drift, so that stability of the statements under these approximations also has to be ensured.

\subsection{Literature}

The well-posedness of SPDEs with monotone, multivalued drift has been investigated by [6] and [4]. The concept of stochastic variational inequalities (SVIs) and a corresponding notion of solution has been established in [7] and [9], and has been applied to generalized stochastic $p$-Laplace equations in [29] and to generalized stochastic fast diffusion equations in [30]. Finally, the existence and uniqueness of SVI solutions to (1.1) follows from a more general well-posedness analysis in [40].

We now aim to give a brief overview on the existing results on ergodicity of stochastic nonlinear diffusions, with a focus on approaches applicable to stochastic (generalized) porous media equations.

In the "classical" approach, e.g. in the monograph [20], the existence of invariant measures to semilinear SPDEs with non-degenerate noise is proven by bounds that imply the tightness of the averaged transition probabilities, allowing to use the Krylov-Bogoliubov theorem. Uniqueness is then relying on the Doob-Khasminskii theorem, using the regularity of the Markov semigroup which can be guaranteed by the strong Feller property and irreducibility. This technique has been considerably improved by [34], using smoothing in form of the asymptotic Feller property, though the scope was still on semilinear equations.

Invariant measures to quasilinear diffusions with additive noise have been initially studied in [18] and [17] on the level of Kolmogorov equations. In [19] (see also the monograph [5]), the strong monotonicity of the porous media operator was exploited, which leads to the existence and uniqueness of invariant measures by strong dissipativity. Further contraction estimates ensuring ergodicity have been obtained via Harnack inequalities (cf. [37,45,46], with explicit estimates for the rate of exponential mixing given in [47]), relying on a non-degeneracy assumption for the noise, and via more careful decay estimates (cf. [39]), treating weakly dissipative drift operators. Stochastic porous media equations with purely multiplicative noise have been treated in [21], using a dissipativity estimate in a weighted $L^{1}$ space. Lower bound techniques as introduced in [35,36] were used by [31] and [32], where generalized porous media equations with discontinuous nonlinearities are analyzed as explained before.

A different approach to the long-time behaviour of solutions to SPDEs is to analyze the existence and the structure of random attractors of random dynamical systems, as e.g. in $[10,15,16,24,27,28]$. A property which has turned out to be very useful in this context is order preservation of trajectories which are driven by the same noise, see, e. g., [1,12,23,25]. A close connection between random attractors and ergodic and mixing properties of random dynamical systems can be obtained in the case of synchronization (see [14]), which is on hand if the random attractor is a singleton. This case has been investigated in, e. g., [12,13,22,23,43].

Last but not least, we mention $[3,8,26]$, where similar equations are considered under multiplicative noise, leading to finite-time absorption of the process into a subcritical region.

\subsection{Structure of the Paper}

After stating the exact setting in the first part of Sect. 2, we state the main result of this article, Theorem 1 at the end of Sect. 2. Section 3 then collects auxiliary results in the natural order of the argumentation, which finally allow to prove Theorem 1. 


\subsection{Notation}

On a bounded open set $\mathcal{O} \subset \mathbb{R}$, we use the classical notation $L^{p}:=L^{p}(\mathcal{O})$ for the Lebesgue space with exponent $p \in[1, \infty]$ with norm $\|\cdot\|_{p}$. We write $H_{0}^{1}:=H_{0}^{1}(\mathcal{O})$ for the Sobolev space of weakly differentiable functions with exponent 2 and zero trace, and its topological dual will be denoted by $H^{-1}$. A bounded linear operator $T: U \rightarrow H$, where $U$ and $H$ are separable Hilbert spaces, is called Hilbert-Schmidt if

$$
\|T\|_{L_{2}(U, H)}:=\sum_{k \in \mathbb{N}}\left\|T e_{k}\right\|_{H}^{2}<\infty
$$

where $\left(e_{k}\right)_{k \in \mathbb{N}}$ is an orthonormal basis of $U$. For a Hilbert space $H, C_{b}(H)$ denotes the space of bounded continuous functions on $H, \mathcal{B}(H)$ denotes the Borel $\sigma$-algebra, and $\mathcal{B}_{b}(H)$ the set of bounded functions $H \rightarrow \mathbb{R}$ which are $\mathcal{B}(H)-\mathcal{B}(\mathbb{R})$-measurable. Multivalued operators on $H$, which arise in this work as subdifferentials of proper, convex and lower-semicontinuous functionals, are mappings $A: H \rightarrow 2^{H}$. We define the domain of $A$ by

$$
D(A):=\{x \in H: A(x) \neq \emptyset\}
$$

and its range by

$$
R(A):=\bigcup_{x \in H} A(x)
$$

For a metric space $V$ and $r>0$, we denote by $B_{r}^{V}$ the open ball with radius $r$ with respect to the corresponding metric. If $V=L^{\infty}$, we use $B_{r}^{\infty}$ for $B_{r}^{L^{\infty}}$. Within term manipulations, the constant $C$ may vary from line to line.

\section{Setting and Main Result}

We consider a one-dimensional open bounded interval $\mathcal{O} \subset \mathbb{R}$ as the underlying domain. For simplicity, set $\mathcal{O}:=(-1,1)$.

Define by $\phi: \mathbb{R} \rightarrow 2^{\mathbb{R}}$ the multi-valued maximal monotone extension of

$$
\mathbb{R} \ni x \mapsto x \mathbf{1}_{\{|x|>1\}},
$$

and let $\psi: \mathbb{R} \rightarrow \mathbb{R}$ be its anti-derivative with $\psi(0)=0$, i. e.

$$
\psi(x)=\frac{1}{2}\left(|x|^{2}-1\right) \mathbf{1}_{\{|x|>1\}} .
$$

Let furthermore $\varphi: H^{-1} \rightarrow[0, \infty]$ be defined as

$$
\varphi(u)= \begin{cases}\int_{\mathcal{O}} \psi(u) \mathrm{d} x & \text { if } u \in L^{2} \\ +\infty & \text { else }\end{cases}
$$

and consider the SPDE

$$
\begin{aligned}
\mathrm{d} X_{t}^{x} & \in-\partial \varphi\left(X_{t}^{x}\right) \mathrm{d} t+B \mathrm{~d} W_{t}, \\
X_{0}^{x} & =x,
\end{aligned}
$$

where $x \in H^{-1}, W$ is an Id-cylindrical Wiener process in some separable Hilbert space $U$, defined on a probability space $(\Omega, \mathcal{F}, \mathbb{P})$ with normal filtration $\left(\mathcal{F}_{t}\right)_{t \in[0, T]}$, and $B \in$ 
$L_{2}\left(U, L^{2}\right)$ is a Hilbert-Schmidt operator. This leads to $B W_{t}$ being a trace-class Wiener process in $L^{2}$, such that there are mutually orthogonal $L^{2}$ functions $\left(\xi_{k}\right)_{k \in \mathbb{N}}$ with

$$
\sum_{k \in \mathbb{N}}\left\|\xi_{k}\right\|_{2}^{2}<\infty
$$

for which

$$
B W_{t}=\sum_{i=1}^{\infty} \beta_{k}(t) \xi_{k},
$$

where $\left(\beta_{k}\right)_{k \in \mathbb{N}}$ are independent one-dimensional standard Brownian motions. Additionally, we impose that there are $m \in \mathbb{N}, c_{1}, \ldots, c_{m} \in \mathbb{R}$ such that

$$
g \in L^{2}, \quad g(x):=\sum_{k=1}^{m} c_{k} \xi_{k}(x)>1 \text { for almost all } x \in \mathcal{O} .
$$

Note that the well-posedness of the SPDE (2.2) has been shown in [40] in the sense of SVIsolutions, identifying $x$ with an almost surely constant random variable $x \in L^{2}\left(\Omega, H^{-1}\right)$. The process constructed there gives rise to a semigroup $\left(P_{t}\right)_{t \geq 0}$ of Markov transition kernels by

$$
P_{t}(x, A)=\mathbb{E} \mathbf{1}_{A}\left(X_{t}^{x}\right) \text { for } x \in H^{-1} \text { and } A \in \mathcal{B}\left(H^{-1}\right),
$$

which will be shown below in Lemma 4 . By a slight abuse of notation, we will denote the induced semigroup on $\mathcal{B}_{b}\left(H^{-1}\right)$ also by $P_{t}$, i.e.

$$
P_{t} f(x)=\int_{H^{-1}} f(y) P_{t}(x, \mathrm{~d} y) \text { for } f \in \mathcal{B}_{b}\left(H^{-1}\right), x \in H^{-1} .
$$

The main result of this article is the following:

Theorem 1 In the setting described above, the semigroup $\left(P_{t}\right)_{t \geq 0}$ admits a unique invariant probability Borel measure $\mu$ on $H^{-1}$, i.e. for all $f \in C_{b}\left(H^{-1}\right)$ we have

$$
\int_{H^{-1}} P_{t} f \mathrm{~d} \mu=\int_{H^{-1}} f \mathrm{~d} \mu \text {. }
$$

We briefly mention the steps of the proof. After we introduce the main approximating object $X^{x, \varepsilon}$ to solutions $X^{x}$ of (2.2), we prove a contraction principle, i.e.

$$
\mathbb{P}\left(\left\|X_{T}^{x}-X_{T}^{y}\right\|_{H^{-1}} \leq\|x-y\|_{H^{-1}}\right)=1 \text { for all } T>0,
$$

which will be needed throughout the remaining proof. The lower bound technique of [35] is then applied in three steps: We first prove that solutions to (2.2) are likely to stay on average close to a ball in $L^{\infty}$, i.e. for $\rho, \delta>0$ there exists an $R>0$ such that for sufficiently large $T>0$

$$
\frac{1}{T} \int_{0}^{T} \mathbb{P}\left(X_{r}^{x} \in C_{\delta}(R)\right) \mathrm{d} r \geq 1-\rho,
$$

where $C_{\delta}(R)$ is the $\delta$-neighbourhood of $B_{R}^{\infty}(0)$ in $H^{-1}$. We then analyze the deterministic equation

$$
\begin{aligned}
\frac{\mathrm{d}}{\mathrm{d} t} u^{ \pm R} & =-\partial \varphi\left(u^{ \pm R}\right)+g \\
u^{ \pm R}(0) & \equiv \pm R
\end{aligned}
$$


which will serve as the control process mentioned above and which converges for large times to a limit $u_{\infty} \in H^{-1}$. Finally, we show that with positive probability, $X^{x}$ behaves "similar" to $u^{ \pm R}$ if $x \in C_{\delta}(R)$, so that together with (2.8) we conclude that for all $x \in H^{-1}, \delta>0$

$$
\liminf _{T \rightarrow \infty} \frac{1}{T} \int_{0}^{T} P_{r}\left(x, B_{2 \delta}^{H^{-1}}\left(u_{\infty}\right)\right) \mathrm{d} r>0,
$$

which implies the existence and uniqueness of an invariant measure by [35, Theorem 1].

\section{Lemmas and Proof}

We recall the following notion from [35]:

Definition 1 We say that a transition semigroup $\left(P_{t}\right)_{t \geq 0}$ on some Hilbert space $H$ has the e-property if the family of functions $\left(P_{t} f\right)_{t \geq 0}$ is equicontinuous at every point $x \in H$ for any bounded and Lipschitz continuous function $f: H \rightarrow \mathbb{R}$.

As mentioned before, the proof of the main theorem relies on the following sufficient condition of [35]:

Proposition 1 (Komorowski-Peszat-Szarek 2010) Let $\left(P_{t}\right)_{t \geq 0}$ be the transition semigroup of a stochastically continuous Markov process taking values on a separable Hilbert space $H$. Assume that $\left(P_{t}\right)_{t \geq 0}$ satisfies the Feller-and the e-property. Furthermore, assume that there exists $z \in H$ such that for every $\delta>0$ and $x \in H$

$$
\liminf _{T \rightarrow \infty} \frac{1}{T} \int_{0}^{T} P_{r}\left(x, B_{\delta}^{H}(z)\right) \mathrm{d} r>0 .
$$

Then the semigroup $\left(P_{t}\right)_{t \geq 0}$ admits a unique invariant probability Borel measure.

Most of the following arguments involve an approximating process, which will be introduced in the following lemmas.

Lemma 1 Let $\phi^{\varepsilon}$ be the Yosida approximation of $\phi$, as introduced in Appendix 1. Let $T>0$ and $x \in L^{2}$, and consider the SPDE

$$
\begin{aligned}
\mathrm{d} X_{t}^{x, \varepsilon} & =\varepsilon \Delta X_{t}^{x, \varepsilon} \mathrm{d} t+\Delta \phi^{\varepsilon}\left(X_{t}^{x, \varepsilon}\right) \mathrm{d} t+B \mathrm{~d} W_{t}, \\
X_{0} & =x .
\end{aligned}
$$

Then, identifying $x$ with a random variable $x \in L^{2}\left(\Omega, L^{2}\right)$ being almost surely constant, (3.2) allows for a unique variational solution $\left(X_{t}^{x, \varepsilon}\right)_{t \in[0, T]}$ in the sense of $[41$, Definition 4.2.1] with respect to the Gelfand triple $L^{2} \hookrightarrow H^{-1} \hookrightarrow\left(L^{2}\right)^{\prime}$. Furthermore, $X^{x, \varepsilon}$ satisfies the regularity estimate

$$
\mathbb{E} \sup _{t \in[0, T]}\left\|X_{t}^{x, \varepsilon}\right\|_{2}^{2}+\varepsilon \mathbb{E} \int_{0}^{T}\left\|X_{r}^{x, \varepsilon}\right\|_{H_{0}^{1}}^{2} \mathrm{~d} r \leq C(T)\left(\mathbb{E}\|x\|_{2}^{2}+1\right)
$$

with a constant $C(T)>0$ independent of $\varepsilon$. For $\left(x_{n}\right)_{n \in \mathbb{N}} \subset L^{2}, x_{n} \rightarrow x$ in $H^{-1}$ for $n \rightarrow \infty$, we have

$$
\lim _{n \rightarrow \infty} \lim _{\varepsilon \rightarrow 0} X^{x_{n}, \varepsilon}=X^{x}
$$


where the limits are taken in $L^{2}\left(\Omega, C\left([0, T], H^{-1}\right)\right)$ and $X^{x}$ is the SVI solution to (2.2). More precisely, the $\varepsilon$-limit is uniform on bounded sets of $L^{2}$ by the estimate

$$
\mathbb{E} \sup _{t \in[0, T]}\left\|X^{y, \varepsilon}-X^{y}\right\|_{H^{-1}}^{2} \leq \varepsilon C(T)\left(\|y\|_{2}^{2}+1\right)
$$

for $y \in L^{2}$, and for the $n$-limit we have

$$
\mathbb{E} \sup _{t \in[0, T]}\left\|X^{x_{n}}-X^{x}\right\|_{H^{-1}}^{2} \leq C(T)\left\|x-x_{n}\right\|_{H^{-1}}^{2} .
$$

Finally, for $x, y \in H^{-1}$ we have

$$
\sup _{t \in[0, T]} \mathbb{E}\left\|X_{t}^{x}-X_{t}^{y}\right\|_{H^{-1}}^{2} \leq C(T)\|x-y\|_{H^{-1}}^{2} .
$$

Proof This becomes clear by [40, Section 4], where the well-posedness for (3.2) goes back to [41, Theorem 4.2.4], always identifying $x$ and $y$ with almost surely constant random variables in the respective spaces. For the quantitative estimates, see especially [40, Equation (4.2)].

Remark 1 We note that if $0<T_{1}<T_{2}<\infty, x \in L^{2}, X^{x, \varepsilon}$ is a solution to (3.2) constructed on $\left[0, T_{1}\right]$ and $Y^{x, \varepsilon}$ is a solution to (3.2) constructed on $\left[0, T_{2}\right]$, then $\left(Y_{t}^{x, \varepsilon}\right)_{t \in\left[0, T_{1}\right]}$ is also a solution to (3.2). By the uniqueness part of [41, Theorem 4.2.4], we have

$$
X_{t}^{x, \varepsilon}=Y_{t}^{x, \varepsilon} \quad \text { for all } t \in\left[0, T_{1}\right]
$$

Consequently, $X_{t}^{x, \varepsilon}$ is consistently defined for all $t \geq 0, x \in H^{-1}$, and the same is true for $X_{t}^{x}$ by (3.4).

From [38, Section 4.3], we recall the following disintegration result.

Lemma 2 The solution to (3.2) is a time-homogeneous Markov process, such that we have

$$
\mathbb{E} f\left(X_{t+s}^{x, \varepsilon}\right)=\mathbb{E}_{\omega_{1}} \mathbb{E}_{\omega_{2}} f\left(X_{t}^{X_{s}^{x, \varepsilon}\left(\omega_{1}\right), \varepsilon}\left(\omega_{2}\right)\right)
$$

for any $f \in \mathcal{B}_{b}\left(L^{2}\right)$ and $t, s>0$.

We need that solutions to (2.2) are almost surely contractive, which will be important in the subsequent analysis.

Lemma 3 Let $x, y \in H^{-1}$ and let $\left(X_{t}^{x}\right)_{t \geq 0}$ and $\left(X_{t}^{y}\right)_{t \geq 0}$ be the SVI solutions to (2.2) with initial value $x$ and $y$, respectively. Then for all $T>0$ we have

$$
\mathbb{P}\left(\left\|X_{T}^{x}-X_{T}^{y}\right\|_{H^{-1}} \leq\|x-y\|_{H^{-1}}\right)=1 .
$$

Proof We first fix $T>0$ for which we want to show the statement.

Step 1: First we prove contractivity on the level of approximate solutions and $x, y \in L^{2}$. For this, let $\left(X_{t}^{x, \varepsilon}\right)_{t \in[0, T]}$ and $\left(X_{t}^{y, \varepsilon}\right)_{t \in[0, T]}$ solve (3.2) with the respective initial value. Let furthermore $Z_{t}:=X_{t}^{x, \varepsilon}-X_{t}^{y, \varepsilon}$, which solves

$$
\begin{aligned}
\mathrm{d} Z_{t} & =\varepsilon \Delta\left(X_{t}^{x, \varepsilon}-X_{t}^{y, \varepsilon}\right) \mathrm{d} t+\left(\Delta \phi^{\varepsilon}\left(X_{t}^{x, \varepsilon}\right)-\Delta \phi^{\varepsilon}\left(X_{t}^{y, \varepsilon}\right)\right) \mathrm{d} t, \\
Z_{0} & =x-y .
\end{aligned}
$$


Then, by Ito's formula (see e.g. [41, Theorem 4.2.5]), and noting that $Z \in H_{0}^{1} \mathbb{P} \otimes \mathrm{d} t$-almost surely by (3.3), we obtain $\mathbb{P}$-almost surely

$$
\begin{aligned}
\left\|Z_{t}\right\|_{H^{-1}}^{2}= & \|x-y\|_{H^{-1}}^{2}+2 \varepsilon \int_{0}^{t}\left\langle\Delta Z_{r}, Z_{r}\right\rangle_{H^{-1}} \mathrm{~d} r \\
& +\int_{0}^{t}\left\langle\Delta \phi^{\varepsilon}\left(X_{r}^{x, \varepsilon}\right)-\Delta \phi^{\varepsilon}\left(X_{r}^{y, \varepsilon}\right), Z_{r}\right\rangle_{H^{-1}} \mathrm{~d} r \\
= & \|x-y\|_{H^{-1}}^{2}-2 \varepsilon \int_{0}^{t}\left\|Z_{r}\right\|_{2}^{2} \mathrm{~d} r \\
& -\int_{0}^{t}\left\langle\phi^{\varepsilon}\left(X_{r}^{x, \varepsilon}\right)-\phi^{\varepsilon}\left(X_{r}^{y, \varepsilon}\right), X_{r}^{x, \varepsilon}-X_{r}^{y, \varepsilon}\right\rangle_{L^{2}} \mathrm{~d} r .
\end{aligned}
$$

The last two terms (the latter because of the monotonicity of $\phi^{\varepsilon}$ ) are negative, which yields

$$
\mathbb{P}\left(\left\|X_{T}^{x, \varepsilon}-X_{T}^{y, \varepsilon}\right\|_{H^{-1}}-\|x-y\|_{H^{-1}}>0\right)=0 .
$$

Step 2: We now turn to SVI solutions to (2.2) with $x, y \in L^{2}$. Note that it is enough to show for arbitrary $n \in \mathbb{N}, \gamma>0$ that

$$
\mathbb{P}\left(\left\|X_{T}^{x}-X_{T}^{y}\right\|_{H^{-1}}-\|x-y\|_{H^{-1}}>\frac{1}{n}\right) \leq \gamma .
$$

To obtain this, choose $\varepsilon$ sufficiently small such that by (3.5)

$$
\max \left\{\mathbb{E}\left\|X_{T}^{x, \varepsilon}-X_{T}^{x}\right\|_{H^{-1}}, \mathbb{E}\left\|X_{T}^{y, \varepsilon}-X_{T}^{y}\right\|_{H^{-1}}\right\}<\frac{\gamma}{4 n},
$$

which yields by Markov's inequality that

$$
\mathbb{P}\left(\left\|X_{T}^{x, \varepsilon}-X_{T}^{x}\right\|_{H^{-1}} \geq \frac{1}{2 n}\right) \leq \frac{\gamma}{2}
$$

and the corresponding statement for $X_{T}^{y}$. Thus together with (3.9) we have

$$
\begin{aligned}
\mathbb{P} & \left(\left\|X_{T}^{x}-X_{T}^{y}\right\|_{H^{-1}}-\|x-y\|_{H^{-1}}>\frac{1}{n}\right) \\
& \leq \mathbb{P}\left(\left\|X_{T}^{x}-X_{T}^{x, \varepsilon}\right\|_{H^{-1}} \geq \frac{1}{2 n}\right)+\mathbb{P}\left(\left\|X_{T}^{y}-X_{T}^{y, \varepsilon}\right\|_{H^{-1}} \geq \frac{1}{2 n}\right) \\
\quad & +\mathbb{P}\left(\left\|X_{T}^{x, \varepsilon}-X_{T}^{y, \varepsilon}\right\|_{H^{-1}}-\|x-y\|_{H^{-1}}>0\right) \\
& \leq \gamma,
\end{aligned}
$$

which yields (3.8) in the case $x, y \in L^{2}$.

Step 3: Finally consider $x, y \in H^{-1}$. By (3.7) we know that for $x, y \in H^{-1}$

$$
\mathbb{E}\left\|X_{T}^{x}-X_{T}^{y}\right\|_{H^{-1}} \leq C\|x-y\|_{H^{-1}} .
$$

In order to confirm (3.10), we choose $\tilde{x}, \tilde{y} \in L^{2}$ in a way that $\left(\|\cdot\|=\|\cdot\|_{H^{-1}}\right)$

$$
\max \{\|x-\tilde{x}\|,\|y-\tilde{y}\|\} \leq \frac{1}{4 n} \text { and } \max \{C\|x-\tilde{x}\|, C\|y-\tilde{y}\|\} \leq \frac{\gamma}{8 n} .
$$

Using

$$
\|x-y\|=\|x-\tilde{x}+\tilde{x}-\tilde{y}+\tilde{y}-y\| \geq\|\tilde{x}-\tilde{y}\|-\|x-\tilde{x}\|-\|y-\tilde{y}\|
$$


and, again by Markov's inequality,

$$
\max \left\{\mathbb{P}\left(\left\|X_{T}^{x}-X_{T}^{\tilde{x}}\right\| \geq \frac{1}{4 n}\right), \mathbb{P}\left(\left\|X_{T}^{y}-X_{T}^{\tilde{y}}\right\| \geq \frac{1}{4 n}\right)\right\} \leq \frac{\gamma}{2},
$$

we compute

$$
\begin{aligned}
& \mathbb{P}\left(\left\|X_{T}^{x}-X_{T}^{y}\right\|_{H^{-1}}-\|x-y\|_{H^{-1}}>\frac{1}{n}\right) \\
& \quad \leq \mathbb{P}\left(\left\|X_{T}^{x}-X_{T}^{\tilde{x}}\right\| \geq \frac{1}{4 n}\right)+\mathbb{P}\left(\left\|X_{T}^{\tilde{x}}-X_{T}^{\tilde{y}}\right\|-\|\tilde{x}-\tilde{y}\|>0\right) \\
& \quad+\mathbb{P}\left(\left\|X_{T}^{\tilde{y}}-X_{T}^{y}\right\| \geq \frac{1}{4 n}\right)+\mathbb{P}\left(\|x-\tilde{x}\| \geq \frac{1}{4 n}\right)+\mathbb{P}\left(\|y-\tilde{y}\| \geq \frac{1}{4 n}\right) \leq \gamma,
\end{aligned}
$$

which finishes the proof.

Lemma 4 The solution to (2.2) gives rise to a semigroup of Markov transition kernels by

$$
P_{t}(x, A)=\mathbb{E} \mathbf{1}_{A}\left(X_{t}^{x}\right) \text { for } x \in H^{-1} \text { and } A \in \mathcal{B}\left(H^{-1}\right) .
$$

The induced semigroup $\left(P_{t}\right)_{t \geq 0}$ on $\mathcal{B}_{b}\left(H^{-1}\right)$, given by

$$
P_{t} f(x)=\int_{H^{-1}} f(y) P_{t}(x, \mathrm{~d} y),
$$

has the Feller-and the e-property. For all $x \in H^{-1}$ and $f \in C_{b}\left(H^{-1}\right)$,

$$
[0, \infty) \ni t \mapsto P_{t} f(x)
$$

is continuous at $t=0$.

Remark 2 The semigroup $\left(P_{t}\right)_{t \geq 0}$ consisting of Markov transition kernels together with the obvious fact

$$
P_{0}(x, A)=\mathbf{1}_{A}(x)
$$

implies that there is a "canonical" Markov process with transition probabilities $\left(P_{t}\right)_{t \geq 0}$ (see e.g. [20, Section 2.2]).

Remark 3 Note that the last statement in Lemma 4 implies the stochastic continuity of $\left(P_{t}\right)_{t \geq 0}$ by [20, Proposition 2.1.1]. By [20, Theorem 2.2.2], the corresponding canonical process is then also stochastically continuous.

Proof (Proof of Lemma 4:) The continuity of (3.11) follows from the construction as an almost surely continuous process, and the Feller property from the contractivity in Lemma 3. In both arguments, the dominated convergence theorem applies due to the continuity and boundedness of the test functions.

To prove the e-property for $\left(P_{t}\right)_{t \geq 0}$, it is sufficient to show that for $f: H^{-1} \rightarrow \mathbb{R}$ bounded and Lipschitz continuous, $P_{t} f(t \geq 0)$ is Lipschitz continuous with Lipschitz constant independent of $t$ and equal to the Lipschitz constant $[f]_{\text {Lip }}$ of $f$. Using Lemma 3 , we compute for $x, y \in H^{-1}$

$$
\begin{aligned}
\left|P_{t} f(x)-P_{t} f(y)\right| & =\left|\mathbb{E}\left[f\left(X_{t}^{x}\right)-f\left(X_{t}^{y}\right)\right]\right| \\
& \leq \mathbb{E}\left|f\left(X_{t}^{x}\right)-f\left(X_{t}^{y}\right)\right| \\
& \leq \mathbb{E}\left[[f]_{\text {Lip }}\left\|X_{t}^{x}-X_{t}^{y}\right\|_{H^{-1}}\right] \\
& \leq[f]_{\text {Lip }}\|x-y\|_{H^{-1}},
\end{aligned}
$$


as required.

We turn to the kernel properties of $P_{t}$ : For $x \in H^{-1}, t \geq 0, P_{t}(x, \cdot)$ is the pushforward measure of $X_{t}^{x}$ and thereby a probability measure. Moreover, let $A \in \mathcal{B}\left(H^{-1}\right)$. Note that the class of all functions $f \in \mathcal{B}_{b}\left(H^{-1}\right)$, for which

$$
H^{-1} \ni x \mapsto P_{t} f(x)
$$

is measurable, is monotone in the sense of [42, Theorem 0.2.2, i) and ii)]. As the family of bounded Lipschitz functions generates the Borel $\sigma$-algebra and is stable under pointwise multiplication,

$$
H^{-1} \ni x \mapsto P_{t} \mathbf{1}_{A}(x)
$$

is proven to be measurable by the monotone class theorem (see e.g. [42, Theorem 0.2.2]), as soon as we show measurability of (3.12) for bounded and Lipschitz continuous $f$. The latter, however, becomes clear by taking into account that $P_{t} f$ is Lipschitz continuous if $f$ is Lipschitz continuous (see the proof of the e-property above).

To establish the semigroup property, we first note that the class of functions $f \in \mathcal{B}_{b}\left(H^{-1}\right)$, for which the semigroup property

$$
P_{t+s} f(x)=P_{s}\left(P_{t} f\right)(x) \text { for all } t, s \geq 0, x \in H^{-1}
$$

is satisfied, is also monotone, so that it is enough to prove the semigroup property for $f$ : $H^{-1} \rightarrow \mathbb{R}$ being bounded and Lipschitz continuous. For such $f$, the claim follows by using the semigroup property for the approximating process $\left(X_{t}^{x_{n}, \varepsilon}\right)_{t \geq 0}$ with $\varepsilon>0, n \in \mathbb{N}$, $\left(x_{n}\right)_{n \in \mathbb{N}} \subset L^{2}, x_{n} \rightarrow x$ for $n \rightarrow \infty$ as stated in Lemma 2, and passing to the limit via Lemmas 1 and 3 .

The following lemma is an energy estimate for the $L^{\infty}$ norm.

Lemma 5 Let $x \in H^{-1}, \delta, \rho>0$ and for $R>0$

$$
C_{\delta}(R):=\left\{u \in H^{-1}: \exists v \in B_{R}^{\infty}(0) \text { such that }\|u-v\|_{H^{-1}}<\delta\right\},
$$

where $B_{R}^{\infty}(0):=\left\{v \in L^{\infty}:\|v\|_{\infty}<R\right\}$. Then there exists $R=R(\rho, x)>3$ such that for all $T>1$ we have

$$
\frac{1}{T} \int_{0}^{T} \mathbb{P}\left(X_{r}^{x} \in C_{\delta}(R)\right) \mathrm{d} r \geq 1-\rho .
$$

for solutions $X^{x}$ to (2.2).

Proof We first consider the approximating solutions from (3.2) with initial value $\tilde{x} \in L^{2}$, for which we know by (3.3) that they are in $H_{0}^{1}, \mathbb{P} \otimes \mathrm{d} t$-almost surely. We choose $\tilde{x}$ in a way that

$$
\|x-\tilde{x}\|_{H^{-1}} \leq \frac{\delta}{2}
$$

Note also that $\phi^{\varepsilon}$ is weakly differentiable for $\varepsilon>0$ and

$$
\left(\phi^{\varepsilon}\right)^{\prime} \geq \frac{1}{2} \mathbf{1}_{\mathbb{R} \backslash[-1,1]}
$$


for $0<\varepsilon<1$ by (B.2). Ito's formula (see e.g. [41, Theorem 4.2.5]) on the Gelfand triple $H_{0}^{1} \hookrightarrow L^{2} \hookrightarrow H^{-1}$ then yields

$$
\begin{aligned}
\left\|X_{t}^{\tilde{x}, \varepsilon}\right\|_{2}^{2}= & \|\tilde{x}\|_{2}^{2}+\int_{0}^{t} 2_{H_{0}^{1}}\left\langle X_{r}^{\tilde{x}, \varepsilon}, \Delta\left(\varepsilon X_{r}^{\tilde{x}, \varepsilon}+\phi^{\varepsilon}\left(X_{r}^{\tilde{x}, \varepsilon}\right)\right)\right\rangle_{H^{-1}} \mathrm{~d} r \\
& +\int_{0}^{t} 2\left\langle X_{r}^{\tilde{x}, \varepsilon}, B \mathrm{~d} W_{r}\right\rangle_{L^{2}}+\int_{0}^{t} 2\|B\|_{L_{2}\left(U, L^{2}\right)}^{2} \mathrm{~d} r .
\end{aligned}
$$

Abbreviating the last two summands by $K$ and using the chain rule for Sobolev functions (see e.g. [49, Theorem 2.1.11]) and (3.16), we obtain

$$
\begin{aligned}
\left\|X_{t}^{\tilde{x}, \varepsilon}\right\|_{2}^{2}= & \|\tilde{x}\|_{2}^{2}-2 \varepsilon \int_{0}^{t}\left\|\nabla X_{r}^{\tilde{x}, \varepsilon}\right\|_{2}^{2} \mathrm{~d} r \\
& -\int_{0}^{t} \int_{\mathcal{O}} 2\left\langle\nabla X_{r}^{\tilde{x}, \varepsilon}, \nabla \phi^{\varepsilon}\left(X_{r}^{\tilde{x}, \varepsilon}\right)\right\rangle \mathrm{d} x \mathrm{~d} r+K \\
\leq & \|\tilde{x}\|_{2}^{2}-2 \int_{0}^{t} \int_{\mathcal{O}}\left(\phi^{\varepsilon}\right)^{\prime}\left(X_{r}^{\tilde{x}, \varepsilon}\right)\left(\nabla X_{r}^{\tilde{x}, \varepsilon}\right)^{2} \mathrm{~d} x \mathrm{~d} r+K \\
\leq & \|\tilde{x}\|_{2}^{2}-\int_{0}^{t} \int_{\mathcal{O}} \mathbf{1}_{\left\{\left|X_{r}^{\tilde{x}, \varepsilon}\right|>1\right\}}\left(\nabla X_{r}^{\tilde{x}, \varepsilon}\right)^{2} \mathrm{~d} x \mathrm{~d} r+K \\
= & \|\tilde{x}\|_{2}^{2}-\int_{0}^{t} \int_{\mathcal{O}}\left(\mathbf{1}_{\left\{\left|X_{r}^{\tilde{x}, \varepsilon}\right|>1\right\}} \nabla X_{r}^{\tilde{x}, \varepsilon}\right)^{2} \mathrm{~d} x \mathrm{~d} r+K .
\end{aligned}
$$

Defining $A \in \operatorname{Lip}(\mathbb{R})$ by

$$
x \mapsto A(x)=\operatorname{sgn}(x)(|x|-1) \mathbf{1}_{\{|x|>1\}},
$$

we see that almost everywhere

$$
A^{\prime}\left(X_{r}^{\tilde{x}, \varepsilon}\right)=\mathbf{1}_{\left\{\left|X_{r}^{\tilde{x}, \varepsilon}\right|>1\right\}}
$$

Thus, using the chain rule for Sobolev functions and the continuous embedding $H_{0}^{1} \hookrightarrow L^{\infty}$, we continue (3.17) by

$$
\begin{aligned}
\left\|X_{t}^{\tilde{x}, \varepsilon}\right\|_{2}^{2} & \leq\|\tilde{x}\|_{2}^{2}-\int_{0}^{t} \int_{\mathcal{O}}\left(\nabla A\left(X_{r}^{\tilde{x}, \varepsilon}\right)\right)^{2} \mathrm{~d} x \mathrm{~d} r+K \\
& \leq\|\tilde{x}\|_{2}^{2}-C \int_{0}^{t}\left\|A\left(X_{r}^{\tilde{x}, \varepsilon}\right)\right\|_{\infty}^{2} \mathrm{~d} r+K \\
& =\|\tilde{x}\|_{2}^{2}-C \int_{0}^{t}\left(\left\|X_{r}^{\tilde{x}, \varepsilon}\right\|_{\infty}-1\right)_{+}^{2} \mathrm{~d} r+K .
\end{aligned}
$$

For the remaining part

$$
K=\int_{0}^{t} 2\left\langle X_{r}^{\tilde{x}, \varepsilon}, B \mathrm{~d} W_{r}\right\rangle_{L^{2}}+\int_{0}^{t} 2\|B\|_{L_{2}\left(U, L^{2}\right)}^{2} \mathrm{~d} r
$$

we notice that the first summand vanishes in expectation and that the second one can be estimated from above by $C t$ by the assumptions on $B$. Thus, taking expectations in (3.18) provides

$$
\mathbb{E} \int_{0}^{t}\left(\left\|X_{r}^{\tilde{x}, \varepsilon}\right\|_{\infty}-1\right)_{+}^{2} \mathrm{~d} r \leq C\left(\|\tilde{x}\|_{2}^{2}+t\right),
$$


where we emphasize that $C$ does not depend on $\varepsilon$. By the Markov inequality, we then use (3.19) to compute

$$
\begin{aligned}
\frac{1}{T} \int_{0}^{T} \mathbb{P}\left(\left(\left\|X_{r}^{\tilde{x}, \varepsilon}\right\|_{\infty}-1\right)_{+}^{2}>R\right) \mathrm{d} r & \leq \frac{1}{T} \int_{0}^{T} \frac{\mathbb{E}\left(\left\|X_{r}^{\tilde{x}, \varepsilon}\right\|_{\infty}-1\right)_{+}^{2}}{R} \mathrm{~d} r \\
& \leq \frac{C}{T R}\left(\|\tilde{x}\|_{2}^{2}+T\right),
\end{aligned}
$$

which for $T>1$ becomes smaller than $\frac{\rho}{2}$ by choosing $R$ large enough, uniformly in $\varepsilon$. For technical reasons, we impose $R>3$ without loss of generality. For $T>1$ fixed, we now choose $\varepsilon$ small enough such that

$$
\mathbb{E} \sup _{t \in[0, T]}\left\|X_{t}^{\tilde{x}}-X_{t}^{\tilde{x}, \varepsilon}\right\|_{H^{-1}} \leq \frac{\rho \delta}{4} .
$$

By Markov's inequality, (3.20) yields

$$
\mathbb{P}\left(\sup _{t \in[0, T]}\left\|X_{t}^{\tilde{x}}-X_{t}^{\tilde{x}, \varepsilon}\right\|_{H^{-1}} \geq \frac{\delta}{2}\right) \leq \frac{\rho}{2} .
$$

By Lemma 3 and (3.15) we have for $t>0$

$$
\left\|X_{t}^{x}-X_{t}^{\tilde{x}}\right\|_{H^{-1}} \leq \frac{\delta}{2} \text { almost surely, }
$$

which we use to conclude for $R$ as chosen above

$$
\begin{aligned}
\frac{1}{T} \int_{0}^{T} \mathbb{P}\left(X_{r}^{x} \in C_{\delta}(R)\right) \mathrm{d} r \\
\quad \geq \frac{1}{T} \int_{0}^{T} \mathbb{P}\left(X_{r}^{\tilde{x}} \in C_{\frac{\delta}{2}}(R) \mathrm{d} r\right. \\
\quad=1-\frac{1}{T} \int_{0}^{T} \mathbb{P}\left(X_{r}^{\tilde{x}} \notin C_{\frac{\delta}{2}}(R)\right) \mathrm{d} r \\
\geq 1-\frac{1}{T} \int_{0}^{T} \mathbb{P}\left(\left\|X_{r}^{\tilde{x}}-X_{r}^{\tilde{x}, \varepsilon}\right\|_{H^{-1}} \geq \frac{\delta}{2} \text { or }\left\|X_{r}^{\tilde{x}, \varepsilon}\right\|_{\infty} \geq R\right) \mathrm{d} r \\
\quad \geq 1-\frac{1}{T} \int_{0}^{T} \mathbb{P}\left(\left\|X_{r}^{\tilde{x}}-X_{r}^{\tilde{x}, \varepsilon}\right\|_{H^{-1}} \geq \frac{\delta}{2}\right) \\
\quad+\mathbb{P}\left(\left\|X_{r}^{\tilde{x}, \varepsilon}\right\|_{\infty} \geq \sqrt{R}+1\right) \mathrm{d} r \\
\geq 1-\frac{\rho}{2}-\frac{1}{T} \int_{0}^{T} \mathbb{P}\left(\left(\left\|X_{r}^{\tilde{x}, \varepsilon}\right\|_{\infty}-1\right)_{+}^{2} \geq R\right) \mathrm{d} r \\
\quad 1-\rho,
\end{aligned}
$$

as required.

We continue with the analysis of the deterministic control process, for which we cite a translated version of [11, Théorème 3.11]. For the definition of weak and strong solutions, see Definition 3. 
Proposition 2 Let $H$ be a Hilbert space and $A: H \supseteq D(A) \rightarrow H$ a maximal monotone operator of the form $A=\partial \varphi$ for some $\varphi: H \rightarrow[0, \infty]$ convex, proper and lower-semicontinuous. Suppose that for all $\alpha \in \mathbb{R}$ the set

$$
M_{\alpha}:=\left\{x \in H: \varphi(x)+\|x\|^{2} \leq \alpha\right\}
$$

is strongly compact. Let $f \in L_{\mathrm{loc}}^{1}([0, \infty) ; H)$ such that $\lim _{t \rightarrow \infty} f(t)=: f_{\infty}$ exists, $f-f_{\infty} \in$ $L^{1}([0, \infty) ; H)$ and $f_{\infty} \in R(\partial \varphi)$. For $x \in \overline{D(\partial \varphi)}$, let $u^{x}$ be a weak solution to

$$
\begin{aligned}
\frac{\mathrm{d}}{\mathrm{d} t} u^{x} & \in-\partial \varphi\left(u^{x}\right)+f, \\
u(0) & =x .
\end{aligned}
$$

Then $\lim _{t \rightarrow \infty} u^{x}(t)=: u_{\infty}$ exists and

$$
f_{\infty} \in \partial \varphi\left(u_{\infty}\right)
$$

Remark 4 Note that existence even of strong solutions to (3.24) is guaranteed by [11, Théorèmes 3.4 and 3.6] for $t \in[0, T], T>0$. By uniqueness, we can extend the solution to $[0, \infty)$, analogous to Remark 1. In particular, for $t>0$ and $x \in \overline{D(\partial \varphi)}$ we have $u^{x}(t) \in D(\partial \varphi)$.

From the definition of $g$ in (2.5), recall especially that $g \in L^{2}$ and $g>1$ almost everywhere in $\mathcal{O}$. For $x \in \overline{D(\partial \varphi)}$, consider the deterministic evolution equation

$$
\begin{aligned}
& \frac{\mathrm{d}}{\mathrm{d} t} u^{x} \in-\partial \varphi\left(u^{x}\right)+g, \\
& u^{x}(0)=x
\end{aligned}
$$

on $H^{-1}$, where $\varphi$ is defined as in (2.1).

Lemma 6 Let $R>1$. For the initial states $x \equiv \pm R$, Proposition 2 can be applied to problem (3.24) by replacing both $f(t)$ and $f_{\infty}$ by $g$. In this case,

$$
u_{\infty}=\left((-\Delta)^{-1} g\right) \vee 1 \text {. }
$$

Proof The functional $\varphi$ as defined in (2.1) is obviously not constantly $\infty$. Furthermore, it is convex and lower-semicontinuous by [2, Proposition 2.10].

In order to verify the compactness of the sets $M_{\alpha}, \alpha \in \mathbb{R}$, as defined in (3.22), we first show that $M_{\alpha}$ is a bounded subset of $L^{2}$. This is obvious for $\alpha \leq 0$ such that we can restrict to $\alpha>0$ in the following. Indeed, if for $u \in H^{-1} \varphi(u) \leq \alpha<\infty$, then $u \in L^{2}$ by (2.1). Then, we compute

$$
\begin{aligned}
\int_{\mathcal{O}} u^{2} \mathrm{~d} x & \leq|\mathcal{O}|+\int_{\{|u| \geq 1\}}(|u|-1+1)^{2} \mathrm{~d} x \\
& \leq|\mathcal{O}|+\int_{\{|u| \geq 1\}}(|u|-1)^{2}+2(|u|-1)+1 \mathrm{~d} x \\
& \leq|\mathcal{O}|+2 \varphi(u)+2|\mathcal{O}|^{\frac{1}{2}}\left(\int_{\{|u| \geq 1\}}(|u|-1)^{2} \mathrm{~d} x\right)^{\frac{1}{2}}+|\mathcal{O}| \\
& \leq 2|\mathcal{O}|+2 \varphi(u)+2 \sqrt{2}|\mathcal{O}|^{\frac{1}{2}} \varphi^{\frac{1}{2}}(u) \leq C(1+\alpha)<\infty .
\end{aligned}
$$


Since the canonical embedding $L^{2} \hookrightarrow H^{-1}$ is compact, it follows that $\overline{M_{\alpha}}$ is compact. As $\varphi$ is lower-semicontinuous, so is $\varphi+\|\cdot\|_{H^{-1}}^{2}$, and thus $M_{\alpha}$ is also closed. Hence, $M_{\alpha}$ is compact, as required.

We recall from [2, Proposition 2.10] that $\partial \varphi$ can be characterized by

$$
\partial \varphi=\left\{\begin{array}{c}
{[u, w] \in\left(H^{-1} \cap L^{1}\right) \times H^{-1}:} \\
w=-\Delta v, v \in H_{0}^{1}, v(x) \in \phi(u(x)) \text { for a. e. } x \in \mathcal{O}
\end{array}\right\},
$$

with

$$
D(\partial \varphi)=\left\{u \in H^{-1} \cap L^{1}: \exists v \in H_{0}^{1} \text { such that } v \in \phi(u) \text { almost everywhere }\right\} .
$$

To show that the constant functions $\pm R$ are elements of $\overline{D(\partial \varphi)}$, we define for $n \in \mathbb{N}$

$$
v_{n}:=n(1-x) \wedge n(x+1) \wedge R \in H_{0}^{1},
$$

and $u_{n}:=v_{n} \vee 1$. We then have $u_{n} \in H^{-1} \cap L^{1}$ and $v_{n} \in \phi\left(u_{n}\right)$, and thus $u_{n} \in D(\partial \varphi)$. Since $u_{n} \rightarrow R$ in $H^{-1}$, we have that the constant function $R \in \overline{D(\partial \varphi)}$. For the constant function with value $-R$, analogous considerations apply.

Finally, to show (3.25), we first prove that

$$
u_{\infty}=\left((-\Delta)^{-1} g\right) \vee 1
$$

satisfies (3.23) with $f_{\infty}$ replaced by $g$. Setting $v:=(-\Delta)^{-1} g$, we have $v \in H_{0}^{1}$, as $g$ was assumed to be in $L^{2} \subset H^{-1}$, and consequently $v \vee 1 \in H^{-1} \cap L^{1}$. Furthermore, $v>0$ almost everywhere by the strong maximum principle (see [33, Theorem 8.19]) and thus $v \in \phi(v \vee 1)$ a.e., such that $v \vee 1 \in D(\partial \varphi)$. Since additionally $g=-\Delta v$, we have $g \in R(\partial \varphi)$ and $g \in \partial \varphi(v \vee 1)$.

We conclude by noticing that (3.26) is the only choice for $u_{\infty}$ such that (3.23) is satisfied. This becomes clear by the strict monotonicity of $\phi \mid \mathbb{R} \backslash(-1,1)$ and the strict positivity of $(-\Delta)^{-1} g$ by the strong maximum principle.

Similarly to Lemma 1 , we define approximations $u^{x, \varepsilon}$ for equation (3.24) by

$$
\begin{aligned}
\frac{\mathrm{d}}{\mathrm{d} t} u_{t}^{x, \varepsilon} & =\varepsilon \Delta u_{t}^{x, \varepsilon}+\Delta \phi^{\varepsilon}\left(u_{t}^{x, \varepsilon}\right)+g \text { for } t \in(0, S], \\
u_{0}^{x, \varepsilon} & =x,
\end{aligned}
$$

where $S>0$ and $g$ still satisfies assumption (2.5). Analogous to the approximation of $X^{x}$, there is a unique variational solution to (3.27), and if $x \in \overline{D(\partial \varphi)} \cap L^{2}$, so that (3.24) has a strong solution, we obtain

$$
\sup _{t \in[0, S]}\left\|u_{t}^{x, \varepsilon}-u_{t}^{x}\right\|_{H^{-1}}^{2} \leq \varepsilon C(S)\left(\|x\|_{2}^{2}+1\right)
$$

analogous to (3.5).

For these approximating deterministic equations, we need order-preservation in the initial value. A partial order on $H^{-1}$ can be defined as follows:

Definition 2 We write $u \leq v$ in $H^{-1}$, if for all $\eta \in H_{0}^{1}, \eta \geq 0$ almost everywhere, one has

$$
u(\eta) \leq v(\eta) .
$$

Lemma 7 Let $u, v, w \in H^{-1}$. Then $u \leq v \leq w$ in $H^{-1}$ implies

$$
\|v\|_{H^{-1}} \leq\|u\|_{H^{-1}}+\|w\|_{H^{-1}} .
$$


Proof For arbitrary $\eta \in H_{0}^{1},\|\eta\|_{H_{0}^{1}} \leq 1$, we compute

$$
\begin{aligned}
v(\eta) & =v(\eta \wedge 0)+v(\eta \vee 0) \\
& =-v(-(\eta \wedge 0))+v(\eta \vee 0) \\
& \leq-u(-(\eta \wedge 0))+w(\eta \vee 0) \\
& =u(\eta \wedge 0)+w(\eta \wedge 0) \\
& \leq\|u\|_{H^{-1}}+\|w\|_{H^{-1}},
\end{aligned}
$$

where for the last step we note that both $\eta \wedge 0$ and $\eta \vee 0$ are $H_{0}^{1}$ functions with norm less than $\eta$ (see e.g. [49, Corollary 2.1.8]).

For the approximate deterministic dynamics governed by (3.27), we then have the following comparison principle:

Lemma 8 Let $x, y \in L^{\infty} \subseteq L^{2}$ and $x \leq y$ almost everywhere, and let $u^{x, \varepsilon}$ and $u^{y, \varepsilon}$ be the solutions to (3.27) with the corresponding initial values. Then

$$
u_{t}^{x, \varepsilon} \leq u_{t}^{y, \varepsilon} \text { in } H^{-1} \text {, for all } t>0 .
$$

Proof Note that $u^{x, \varepsilon}$ for $x \in L^{\infty}$ is also a weak solution in the sense of [44, Chapter 5] with $\Phi=\varepsilon \mathrm{Id}+\phi^{\varepsilon}$. By [44, Theorem 5.7], the claimed comparison principle is satisfied.

Corollary 1 Let $R>0$. As a consequence of Lemmas 7 and 8, we have for $x \in L^{\infty},\|x\|_{\infty} \leq R$ and arbitrary $u \in H^{-1}$

$$
\left\|u_{t}^{x, \varepsilon}-u\right\|_{H^{-1}} \leq\left\|u_{t}^{R, \varepsilon}-u\right\|_{H^{-1}}+\left\|u_{t}^{-R, \varepsilon}-u\right\|_{H^{-1}} \text { for } t \geq 0 .
$$

Proof It is enough to read off Definition 2 that $-R \leq x \leq R$ almost everywhere implies $-R \leq x \leq R$ in $H^{-1}$, and that the order is invariant under translation by a fixed element of $H^{-1}$.

We now compare the approximations $u^{x, \varepsilon}$ to the solution of the stochastic equation (3.2), with a noise conditioned on suitable events.

Lemma 9 Let $R, S>0,0<\beta \leq 1, x \in L^{\infty},\|x\|_{\infty} \leq R$ and let $u^{x, \varepsilon}$ be the solution to (3.27). Furthermore, let $X^{x, \varepsilon}$ be the solution to (3.2) up to time $S$ with the same initial condition x. Assume that

$$
\sup _{t \in[0, S]}\left\|W_{t}^{B}-\operatorname{tg}\right\|_{2} \leq \beta
$$

where for simplicity we write $W_{t}^{B}=B W_{t}$. Then for $0<\varepsilon \leq 1$ we have

$$
\left\|X_{S}^{x, \varepsilon}-u_{S}^{x, \varepsilon}\right\|_{H^{-1}} \leq C(R, S) \beta .
$$

Proof We consider the transformed processes

$$
\begin{aligned}
Y_{t}^{x, \varepsilon} & =X_{t}^{x, \varepsilon}-W_{t}^{B} \quad \text { and } \\
v_{t}^{x, \varepsilon} & =u_{t}^{x, \varepsilon}-\operatorname{tg},
\end{aligned}
$$

so that by

$$
\left\|X_{S}^{x, \varepsilon}-u_{S}^{x, \varepsilon}\right\|_{H^{-1}} \leq\left\|Y_{S}^{x, \varepsilon}-v_{S}^{x, \varepsilon}\right\|_{H^{-1}}+\left\|W_{S}^{B}-S g\right\|_{H^{-1}},
$$


we can focus on $\left\|Y_{S}^{x, \varepsilon}-v_{S}^{x, \varepsilon}\right\|_{H^{-1}}^{2}$ using (3.29) and the continuity of the embedding $L^{2} \hookrightarrow$ $H^{-1}$. For the following equalities, recall that $X^{x, \varepsilon} \in H_{0}^{1} \mathbb{P} \otimes \mathrm{d} t$-almost everywhere due to (3.3) and $u_{r}^{x, \varepsilon} \in H_{0}^{1}$ for almost every $r \in[0, S]$ by [44, Theorem 5.7]. Thus,

$$
\begin{gathered}
\varepsilon X_{r}^{x, \varepsilon}+\phi^{\varepsilon}\left(X_{r}^{x, \varepsilon}\right) \in H_{0}^{1} \mathbb{P} \otimes \mathrm{d} t-\text { a. e. } \\
\text { and } \varepsilon u_{r}^{x, \varepsilon}+\phi^{\varepsilon}\left(u_{r}^{x, \varepsilon}\right) \in H_{0}^{1} \text { for a. e. } r \in[0, S],
\end{gathered}
$$

by the Lipschitz continuity of $\phi^{\varepsilon}$ and the chain rule for Sobolev functions (e.g. [49, Theorem 2.1.11]), which allows to write

$$
\begin{aligned}
& \frac{1}{2}\left\|Y_{S}^{x, \varepsilon}-v_{S}^{x, \varepsilon}\right\|_{H^{-1}}^{2} \\
& =\int_{0}^{S}\left\langle Y_{r}^{x, \varepsilon}-v_{r}^{x, \varepsilon}, \Delta\left(\varepsilon X_{r}^{x, \varepsilon}+\phi^{\varepsilon}\left(X_{r}^{x, \varepsilon}\right)\right)-\Delta\left(\varepsilon u_{r}^{x, \varepsilon}+\phi^{\varepsilon}\left(u_{r}^{x, \varepsilon}\right)\right)\right\rangle_{H^{-1}} \mathrm{~d} r \\
& =-\int_{0}^{S}\left\langle Y_{r}^{x, \varepsilon}-v_{r}^{x, \varepsilon}, \varepsilon X_{r}^{x, \varepsilon}+\phi^{\varepsilon}\left(X_{r}^{x, \varepsilon}\right)-\left(\varepsilon u_{r}^{x, \varepsilon}+\phi^{\varepsilon}\left(u_{r}^{x, \varepsilon}\right)\right)\right\rangle_{L^{2}} \mathrm{~d} r \\
& =-\int_{0}^{S}\left\langle Y_{r}^{x, \varepsilon}+W_{r}^{B}-\left(v_{r}^{x, \varepsilon}+r g\right), \varepsilon\left(Y_{r}^{x, \varepsilon}+W_{r}^{B}-\left(v_{r}^{x, \varepsilon}+r g\right)\right)\right\rangle_{L^{2}} \mathrm{~d} r \\
& -\int_{0}^{S}\left\langle Y_{r}^{x, \varepsilon}+W_{r}^{B}-\left(v_{r}^{x, \varepsilon}+r g\right), \phi^{\varepsilon}\left(Y_{r}^{x, \varepsilon}+W_{r}^{B}\right)-\phi^{\varepsilon}\left(v_{r}^{x, \varepsilon}+r g\right)\right\rangle_{L^{2}} \mathrm{~d} r \\
& +\int_{0}^{S}\left\langle W_{r}^{B}-r g\right. \\
& \left.\varepsilon\left(Y_{r}^{x, \varepsilon}+W_{r}^{B}-\left(v_{r}^{x, \varepsilon}+r g\right)\right)+\phi^{\varepsilon}\left(Y_{r}^{x, \varepsilon}+W_{r}^{B}\right)-\phi^{\varepsilon}\left(v_{r}^{x, \varepsilon}+r g\right)\right\rangle_{L^{2}} \mathrm{~d} r \\
& \leq \int_{0}^{S}\left\|W_{r}^{B}-r g\right\|_{2} \\
& \times\left\|\varepsilon\left(Y_{r}^{x, \varepsilon}+W_{r}^{B}\right)+\phi^{\varepsilon}\left(Y_{r}^{x, \varepsilon}+W_{r}^{B}\right)-\varepsilon\left(v_{r}^{x, \varepsilon}+r g\right)-\phi^{\varepsilon}\left(v_{r}^{x, \varepsilon}+r g\right)\right\|_{2} \mathrm{~d} r \\
& \leq\left(\int_{0}^{S}\left\|W_{r}^{B}-r g\right\|_{2}^{2} \mathrm{~d} r\right)^{\frac{1}{2}} \\
& \times\left(\int _ { 0 } ^ { S } \left(\varepsilon\left\|Y_{r}^{x, \varepsilon}+W_{r}^{B}\right\|_{2}+\left\|\phi^{\varepsilon}\left(Y_{r}^{x, \varepsilon}+W_{r}^{B}\right)\right\|_{2}\right.\right. \\
& \left.\left.+\varepsilon\left\|v_{r}^{x, \varepsilon}+r g\right\|_{2}+\left\|\phi^{\varepsilon}\left(v_{r}^{x, \varepsilon}+r g\right)\right\|_{2}\right)^{2} \mathrm{~d} r\right)^{\frac{1}{2}} \\
& \leq S^{\frac{1}{2}} \beta\left(4 \int_{0}^{S} \varepsilon^{2}\left\|Y_{r}^{x, \varepsilon}+W_{r}^{B}\right\|_{2}^{2}+\left\|\phi^{\varepsilon}\left(Y_{r}^{x, \varepsilon}+W_{r}^{B}\right)\right\|_{2}^{2}\right. \\
& \left.+\varepsilon^{2}\left\|v_{r}^{x, \varepsilon}+r g\right\|_{2}^{2}+\left\|\phi^{\varepsilon}\left(v_{r}^{x, \varepsilon}+r g\right)\right\|_{2}^{2} \mathrm{~d} r\right)^{\frac{1}{2}} .
\end{aligned}
$$

Note that the monotonicity of $\phi^{\varepsilon}$ has been used for the first inequality. It remains to show that the last factor can be bounded in terms of $R$ and $S$ uniformly in $\beta \leq 1$. 
To see this boundedness, first notice by (B.2) in Appendix 1 that $\left|\phi^{\varepsilon}(x)\right| \leq|x|$ for all $x \in \mathbb{R}, \varepsilon>0$, so that it is enough to prove suitable bounds on

$$
\int_{0}^{S}\left\|Y_{r}^{x, \varepsilon}+W_{r}^{B}\right\|_{2}^{2} \mathrm{~d} r \text { and } \int_{0}^{S}\left\|v_{r}^{x, \varepsilon}+r g\right\|_{2}^{2} \mathrm{~d} r .
$$

To this end, we compute

$$
\frac{1}{2}\left\|Y_{S}^{x, \varepsilon}\right\|_{H^{-1}}^{2}=\|x\|_{H^{-1}}^{2}+\int_{0}^{S}\left\langle\varepsilon \Delta\left(X_{r}^{x, \varepsilon}\right)+\Delta \phi^{\varepsilon}\left(X_{r}^{x, \varepsilon}\right), Y_{r}^{x, \varepsilon}\right\rangle_{H^{-1}} \mathrm{~d} r
$$

by (3.3), and further, noting $Y_{r}^{x, \varepsilon} \in L^{2}$ by (3.3) and (2.4),

$$
\begin{aligned}
(3.31)= & \|x\|_{H^{-1}}^{2}-\int_{0}^{S}\left\langle\varepsilon X_{r}^{x, \varepsilon}+\phi^{\varepsilon}\left(X_{r}^{x, \varepsilon}\right), Y_{r}^{x, \varepsilon}\right\rangle_{L^{2}} \mathrm{~d} r \\
= & \|x\|_{H^{-1}}^{2}-\int_{0}^{S}\left\langle\varepsilon\left(Y_{r}^{x, \varepsilon}+W_{r}^{B}\right)+\phi^{\varepsilon}\left(Y_{r}^{x, \varepsilon}+W_{r}^{B}\right), Y_{r}^{x, \varepsilon}+W_{r}^{B}\right\rangle_{L^{2}} \mathrm{~d} r \\
& +\int_{0}^{S}\left\langle\varepsilon\left(Y_{r}^{x, \varepsilon}+W_{r}^{B}\right)+\phi^{\varepsilon}\left(Y_{r}^{x, \varepsilon}+W_{r}^{B}\right), W_{r}^{B}\right\rangle_{L^{2}} \mathrm{~d} r .
\end{aligned}
$$

From (B.3) in Appendix 1, we obtain the lower bound $\left|\phi^{\varepsilon}(x)\right| \geq \frac{1}{2}|x|$ for $|x| \geq 1+\varepsilon$ and $\varepsilon \leq 1$, so that for $u \in L^{2}$ we have the estimate

$$
\|u\|_{2}^{2} \leq \int_{\{|u| \geq 1+\varepsilon\}} 2 u \phi^{\varepsilon}(u) \mathrm{d} x+4|\mathcal{O}| \leq 2\left\langle u,\left.\phi^{\varepsilon}(u)\right|_{L^{2}}+4|\mathcal{O}| .\right.
$$

Using (3.33) and Young's inequality for the last two summands, once weighted by $\frac{1}{2}$, we continue by

$$
\begin{aligned}
(3.32) \leq & \|x\|_{H^{-1}}^{2}-\int_{0}^{S} \varepsilon\left\|Y_{r}^{x, \varepsilon}+W_{r}^{B}\right\|_{2}^{2}+\frac{1}{2}\left\|Y_{r}^{x, \varepsilon}+W_{r}^{B}\right\|_{2}^{2}-C \mathrm{~d} r \\
& +\int_{0}^{S} \frac{\varepsilon}{2}\left\|Y_{r}^{x, \varepsilon}+W_{r}^{B}\right\|_{2}^{2}+\frac{\varepsilon}{2}\left\|W_{r}^{B}\right\|_{2}^{2}+\frac{1}{4}\left\|\phi^{\varepsilon}\left(Y_{r}^{x, \varepsilon}+W_{r}^{B}\right)\right\|_{2}^{2}+\left\|W_{r}^{B}\right\|_{2}^{2} \mathrm{~d} r \\
\leq & \|x\|_{H^{-1}}^{2}-\frac{1}{4} \int_{0}^{S}\left\|Y_{r}^{x, \varepsilon}+W_{r}^{B}\right\|_{2}^{2} \mathrm{~d} r+\frac{3}{2} \int_{0}^{S}\left\|W_{r}^{B}\right\|_{2}^{2}+C \mathrm{~d} r .
\end{aligned}
$$

By (3.29), assumption (2.5) and $\beta \leq 1$, we have for $r \in[0, S]$

$$
\left\|W_{r}^{B}\right\|_{2} \leq\left\|W_{r}^{B}-r g\right\|_{2}+\|r g\|_{2} \leq \beta+S\|g\|_{2} \leq C(S),
$$

such that (3.34) yields, by dropping the left-hand side and relabelling the constants,

$$
\int_{0}^{S}\left\|Y_{r}^{x, \varepsilon}+W_{r}^{B}\right\|_{2}^{2} \mathrm{~d} r \leq 4 C\left(S,\|x\|_{H^{-1}}^{2}\right) .
$$

To obtain a bound that only depends on $S$ and $R$, note that $x \in L^{\infty},\|x\|_{\infty} \leq R$ by assumption, such that

$$
\|x\|_{H^{-1}} \leq C\|x\|_{2} \leq 2 C|\mathcal{O}|^{\frac{1}{2}} R
$$

which, together with (3.35), yields the desired bound. A similar estimate for $\int_{0}^{S}\left\|v_{r}^{x, \varepsilon}+r g\right\|_{2}^{2} \mathrm{~d} r$ can be obtained by analogous computations.

We need to ensure that (3.29) is realized for each $\beta>0$ with non-zero probability. 
Lemma 10 As in (2.4) we denote

$$
W_{t}^{B}=B W_{t}=\sum_{i=1}^{\infty} \beta_{k}(t) \xi_{k}
$$

with $\sum_{k \in \mathbb{N}}\left\|\xi_{k}\right\|_{2}^{2}<\infty$. Let $g$ and $m$ be defined as in (2.5), and let the degeneracy assumption on $\left(\xi_{k}\right)_{k \in \mathbb{N}}$ in (2.5) be satisfied. Then for all $S \geq 0, \beta>0$ we have

$$
\mathbb{P}\left(\sup _{t \in[0, S]}\left\|W_{t}^{B}-\operatorname{tg}\right\|_{2}^{2} \leq \beta\right)>0 .
$$

Proof We use the orthogonality of $\left(\xi_{k}\right)_{k \in \mathbb{N}}$ to write, for $m^{*}>m$,

$$
\begin{aligned}
& \left\|W_{t}^{B}-t g\right\|_{2}^{2} \\
& =\left\|\sum_{k=1}^{m} \xi_{k}\left(\beta_{k}(t)-t c_{k}\right)\right\|_{2}^{2}+\left\|\sum_{k=m+1}^{m^{*}} \xi_{k} \beta_{k}(t)\right\|_{2}^{2}+\left\|\sum_{k=m^{*}+1}^{\infty} \xi_{k} \beta_{k}(t)\right\|_{2}^{2} \\
& =\sum_{k=1}^{m}\left\|\xi_{k}\right\|_{2}^{2}\left|\beta_{k}(t)-t c_{k}\right|^{2}+\sum_{k=m+1}^{m^{*}}\left\|\xi_{k}\right\|_{2}^{2}\left|\beta_{k}(t)\right|^{2}+\sum_{k=m^{*}+1}^{\infty}\left\|\xi_{k}\right\|_{2}^{2}\left|\beta_{k}(t)\right|^{2} .
\end{aligned}
$$

For the first term, we note that the event

$$
\max _{k \in\{1, \ldots, m\}} \sup _{t \in[0, S]}\left|\beta_{k}(t)-c_{k} t\right|^{2} \leq \frac{\beta}{3 \sum_{k=1}^{m}\left\|\xi_{k}\right\|_{2}}
$$

has positive probability by the following reasoning: As the $\left(\beta_{k}\right)_{k=1}^{m}$ are independent, it is enough to show for each $k \in\{1, \ldots, m\}$ that

$$
\mathbb{P}\left(\sup _{t \in[0, S]}\left|\beta_{k}(t)-c_{k} t\right| \leq \varepsilon\right)>0
$$

for any fixed $S>0, \varepsilon>0$. To see this, note that $\beta_{k}(t)-c_{k} t$ is again a standard Brownian motion with respect to some probability measure $\mathbb{P}_{Q}$, which is absolutely continuous with respect to $\mathbb{P}$ by Girsanov's theorem. From [26, Lemma B.1], we obtain for a standard Brownian motion $\beta_{1}$ that

$$
\mathbb{P}\left(\sup _{t \in[0, S]}\left|\beta_{1}(t)\right| \leq \epsilon\right)>0,
$$

which is equivalent to

$$
\mathbb{P}_{Q}\left(\sup _{t \in[0, S]}\left|\beta_{k}(t)-c_{k} t\right| \leq \varepsilon\right)>0 .
$$

Absolute continuity then yields (3.38). For the third term in (3.36), we compute

$$
\begin{aligned}
\mathbb{E} \sup _{t \in[0, S]} \sum_{k>m^{*}}\left|\beta_{k}(t)\right|^{2}\left\|\xi_{k}\right\|_{2}^{2} & \leq \sum_{k>m^{*}}\left\|\xi_{k}\right\|_{2}^{2} \mathbb{E} \sup _{t \in[0, S]}\left|\beta_{k}(t)\right|^{2} \\
& \leq 4 S \sum_{k>m^{*}}\left\|\xi_{k}\right\|_{2}^{2}=: R\left(m^{*}\right) \searrow 0
\end{aligned}
$$


for $m^{*} \rightarrow \infty$, where we used the squared version of the Burkholder-Davis-Gundy inequality. Choosing $m^{*}$ so large that $R\left(m^{*}\right) \leq \frac{\beta}{3}$ we obtain

$$
\mathbb{P}\left(\sup _{t \in[0, S]} \sum_{k>m^{*}}\left\|\xi_{k}\right\|_{2}^{2}\left|\beta_{k}(t)\right|^{2} \leq \frac{\beta}{3}\right) \geq 1-\frac{R\left(m^{*}\right)}{\frac{\beta}{3}}>0 .
$$

Having chosen $m^{*}$ in this way, we can now conclude by (3.39) that also for the second term of (3.36) we have

$$
\mathbb{P}\left(\sup _{t \in[0, S]} \sum_{k=m+1}^{m^{*}}\left\|\xi_{k}\right\|_{2}^{2}\left|\beta_{k}(t)\right|^{2} \leq \frac{\beta}{3}\right)>0,
$$

which proves the claim by independence.

The following lemma combines all results up to now.

Lemma 11 Let $\delta>0, R>1$ and let $g \in L^{2}$ satisfy assumption (2.5). Recall $u_{\infty}$ from Lemma 6 as the long-time limit of solutions $u^{R}, u^{-R}$ to (3.24). Then there exist $\gamma, S>0$ such that for every initial value $x \in C_{\delta}(R)$, where $C_{\delta}(R)$ is the $\delta$-neighbourhood of $B_{R}^{\infty}(0)$ in $H^{-1}$, we have

$$
\mathbb{P}\left(\left\|X_{S}^{x}-u_{\infty}\right\|_{H^{-1}}<2 \delta\right) \geq \gamma
$$

Proof Recall that $u^{R}, u^{-R}$ are well-defined by Remark 4 and Lemma 6. According to Lemma 6 , we can choose $S>0$ such that we have

$$
\max \left\{\left\|u^{R}(t)-u_{\infty}\right\|_{H^{-1}},\left\|u^{-R}(t)-u_{\infty}\right\|_{H^{-1}}\right\} \leq \frac{\delta}{8} \quad \text { for all } t \geq S .
$$

Let $u^{x, \varepsilon}$ be defined as in Lemma 9. As shown there, we can choose $0<\beta \leq 1$ such that

$$
\sup _{t \in[0, S]}\left\|W_{t}^{B}-\operatorname{tg}\right\|_{2} \leq \beta \text { implies }\left\|X_{S}^{x, \varepsilon}-u_{S}^{x, \varepsilon}\right\|_{H^{-1}}<\frac{\delta}{4},
$$

uniformly for all $\varepsilon \in(0,1], x \in B_{R}^{\infty}(0)$. We then define

$$
\gamma:=\frac{2}{3} \mathbb{P}\left(\sup _{t \in[0, S]}\left\|W_{t}^{B}-\operatorname{tg}\right\|_{2}^{2} \leq \beta\right),
$$

which is strictly positive by Lemma 10 . We then choose $\varepsilon \in(0,1]$ small enough such that for $u^{R, \varepsilon}$ and $u^{-R, \varepsilon}$ as in (3.27) we have

$$
\max \left\{\left\|u_{S}^{R, \varepsilon}-u_{S}^{R}\right\|_{H^{-1}},\left\|u_{S}^{-R, \varepsilon}-u_{S}^{-R}\right\|_{H^{-1}}\right\} \leq \frac{\delta}{8},
$$

which is possible by (3.28), and such that

$$
\mathbb{E} \sup _{r \in[0, S]}\left\|X_{r}^{x, \varepsilon}-X_{r}^{x}\right\|_{H^{-1}} \leq \frac{\gamma \delta}{8}
$$

is satisfied uniformly for $x \in B_{R}^{\infty}(0)$ by (3.5) (note that the squared form in (3.5) is a stronger statement than needed for (3.44) by Jensen's inequality). For every $x \in B_{R}^{\infty}(0)$, (3.44) implies

$$
\mathbb{P}\left(\left\|X_{S}^{x}-X_{S}^{x, \varepsilon}\right\|_{H^{-1}} \leq \frac{\delta}{4}\right) \geq 1-\frac{\gamma}{2}
$$


and Corollary 1, (3.40) and (3.43) yield

$$
\begin{aligned}
\left\|u_{S}^{x, \varepsilon}-u_{\infty}\right\|_{H^{-1}} \leq & \left\|u_{S}^{R, \varepsilon}-u_{\infty}\right\|_{H^{-1}}+\left\|u_{S}^{-R, \varepsilon}-u_{\infty}\right\|_{H^{-1}} \\
\leq & \left\|u_{S}^{R, \varepsilon}-u_{S}^{R}\right\|_{H^{-1}}+\left\|u_{S}^{R}-u_{\infty}\right\|_{H^{-1}} \\
& +\left\|u_{S}^{-R, \varepsilon}-u_{S}^{-R}\right\|_{H^{-1}}+\left\|u_{S}^{-R}-u_{\infty}\right\|_{H^{-1}} \\
\leq & 4 \frac{\delta}{8}=\frac{\delta}{2} .
\end{aligned}
$$

Hence, still for $x \in B_{R}^{\infty}(0)$, we conclude,

$$
\begin{aligned}
& \mathbb{P}\left(\left\|X_{S}^{x}-u_{\infty}\right\|_{H^{-1}}<\delta\right) \\
& \quad \geq \mathbb{P}\left(\left\|X_{S}^{x}-X_{S}^{x, \varepsilon}\right\|_{H^{-1}}<\frac{\delta}{4} \text { and }\left\|X_{S}^{x, \varepsilon}-u_{S}^{x, \varepsilon}\right\|_{H^{-1}}<\frac{\delta}{4}\right) \\
& \quad=1-\mathbb{P}\left(\left\|X_{S}^{x}-X_{S}^{x, \varepsilon}\right\|_{H^{-1}} \geq \frac{\delta}{4} \text { or }\left\|X_{S}^{x, \varepsilon}-u_{S}^{x, \varepsilon}\right\|_{H^{-1}} \geq \frac{\delta}{4}\right) \\
& \quad \geq 1-\mathbb{P}\left(\left\|X_{S}^{x}-X_{S}^{x, \varepsilon}\right\|_{H^{-1}} \geq \frac{\delta}{4}\right)-\mathbb{P}\left(\left\|X_{S}^{x, \varepsilon}-u_{S}^{x, \varepsilon}\right\|_{H^{-1}} \geq \frac{\delta}{4}\right) \\
& \quad \geq \mathbb{P}\left(\left\|X_{S}^{x, \varepsilon}-u_{S}^{x, \varepsilon}\right\|_{H^{-1}}<\frac{\delta}{4}\right)-\frac{\gamma}{2} \\
& \quad \geq \mathbb{P}\left(\sup _{t \in[0, S]}\left\|W_{t}^{B}-\operatorname{tg}\right\|_{2}^{2} \leq \beta\right)-\frac{\gamma}{2}=\gamma .
\end{aligned}
$$

The claim for $x \in C_{\delta}(R)$ follows immediately by Lemma 3 .

Proof (Proof of Theorem 1) Lemma 4, Remark 2 and Remark 3 prove all requirements of Proposition 1 except (3.1). To see this remaining statement, we estimate for $0<\rho<1$ and $R(\rho, x)$ given in Lemma 5

$$
\begin{aligned}
& \liminf _{T \rightarrow \infty} \frac{1}{T} \int_{0}^{T} P_{r}\left(x, B_{2 \delta}^{H^{-1}}\left(u_{\infty}\right)\right) \mathrm{d} r \\
& \quad=\liminf _{T \rightarrow \infty} \frac{1}{T} \int_{0}^{T} P_{r+S}\left(x, B_{2 \delta}^{H^{-1}}\left(u_{\infty}\right)\right) \mathrm{d} r \\
& \quad=\liminf _{T \rightarrow \infty} \frac{1}{T} \int_{0}^{T} \int_{H^{-1}} P_{S}\left(y, B_{2 \delta}^{H^{-1}}\left(u_{\infty}\right)\right) P_{r}(x, \mathrm{~d} y) \mathrm{d} r \\
& \quad \geq \liminf _{T \rightarrow \infty} \frac{1}{T} \int_{0}^{T} \int_{C_{\delta}(R(\rho, x))} P_{S}\left(y, B_{2 \delta}^{H^{-1}}\left(u_{\infty}\right)\right) P_{r}(x, \mathrm{~d} y) \mathrm{d} r \\
& \quad \geq \gamma \liminf _{T \rightarrow \infty} \frac{1}{T} \int_{0}^{T} P_{r}\left(x, C_{\delta}(R(\rho, x)) \mathrm{d} r>\gamma(1-\rho)>0,\right.
\end{aligned}
$$

where we used the semigroup property of $\left(P_{t}\right)_{t \geq 0}$, Lemma 11 and Lemma 5. The result then follows by Proposition 1.

Acknowledgements I would like to thank my advisor Benjamin Gess for his support throughout my work on this article. Support by the International Max Planck Research School in Leipzig and by the "Cusanuswerk Bischöfliche Studienförderung" is gratefully acknowledged. Open Access funding enabled and organized by Projekt DEAL. 
Funding Open Access funding enabled and organized by Projekt DEAL.

Open Access This article is licensed under a Creative Commons Attribution 4.0 International License, which permits use, sharing, adaptation, distribution and reproduction in any medium or format, as long as you give appropriate credit to the original author(s) and the source, provide a link to the Creative Commons licence, and indicate if changes were made. The images or other third party material in this article are included in the article's Creative Commons licence, unless indicated otherwise in a credit line to the material. If material is not included in the article's Creative Commons licence and your intended use is not permitted by statutory regulation or exceeds the permitted use, you will need to obtain permission directly from the copyright holder. To view a copy of this licence, visit http://creativecommons.org/licenses/by/4.0/.

\section{A. Solutions to Monotone Evolution Equations}

For the reader's convenience, we cite and translate [11, Definition 3.1]:

Definition 3 Let $H$ be a Hilbert space, $f \in L^{1}([0, T] ; H), A: H \supseteq D(A) \rightarrow H$ a maximal monotone operator. A function $u \in C\left([0, T] ; H^{-1}\right)$ is called a strong solution to

$$
\frac{\mathrm{d}}{\mathrm{d} t} u \in-A u+f
$$

if $u$ is absolutely continuous on compact subsets of $(0, T)$ (which implies that $u$ is differentiable almost everywhere in $(0, T))$ and for almost all $t \in(0, T)$

$$
u(t) \in D(A)
$$

and

$$
\frac{\mathrm{d} u}{\mathrm{~d} t}(t) \in-A u(t)+f(t) .
$$

We call $u \in C\left([0, T] ; H^{-1}\right)$ a weak solution to (A.1) if there are sequences $f_{n} \in$ $L^{1}([0, T] ; H)$ and $u_{n} \in C([0, T] ; H)(n \in \mathbb{N})$ such that $u_{n}$ is a strong solution of the equation

$$
\frac{\mathrm{d}}{\mathrm{d} t} u_{n} \in-A u_{n}+f_{n}
$$

$f_{n} \rightarrow f$ in $L^{1}([0, T] ; H)$ and $u_{n} \rightarrow u$ uniformly in $[0, T]$ for $n \rightarrow \infty$.

Remark 5 We observe that each strong solution is also a weak solution.

\section{B. Yosida Approximation for the Specific Function $\phi$}

Recall from Sect. 2 that the multivalued function $\phi: \mathbb{R} \rightarrow \mathbb{R}$ is defined as the maximal monotone extension of

$$
\mathbb{R} \ni x \mapsto x \mathbf{1}_{\{|x|>1\}} .
$$

We want to explicitly calculate its resolvent function $R^{\varepsilon}: \mathbb{R} \rightarrow \mathbb{R}$ and its Yosida approximation $\phi^{\varepsilon}: \mathbb{R} \rightarrow \mathbb{R}$. For theoretical details, see [30, Appendix C].

The resolvent $R^{\varepsilon}(x)$ is defined as the solution $s$ to

$$
s+\varepsilon \phi(s) \ni x .
$$


Note that (B.1) has exactly one solution by the maximal monotonicity of $\phi$. For $x \in[-1,1]$ we have

$$
0 \in \phi(x)
$$

thus (B.1) is solved by $s=x$. Consequently $R^{\varepsilon}(x)=x$.

For $x \in(1,1+\varepsilon]$ we have

$$
\frac{x-1}{\varepsilon} \in[0,1]=\phi(1)
$$

Thus, $s=1$ solves the equation by

$$
x=1+\varepsilon \frac{x-1}{\varepsilon} \in 1+\varepsilon \phi(1),
$$

which yields $R^{\varepsilon}(x)=1$. If $x \in[-1-\varepsilon, 1)$, the same argument yields $R^{\varepsilon}(x)=-1$.

For $|x|>1+\varepsilon$, we have $\left|\frac{x}{1+\varepsilon}\right|>1$ such that

$$
x=\frac{x}{1+\varepsilon}+\varepsilon \frac{x}{1+\varepsilon} \in \frac{x}{1+\varepsilon}+\varepsilon \phi\left(\frac{x}{1+\varepsilon}\right),
$$

yielding $R^{\varepsilon}(x)=\frac{x}{1+\varepsilon}$. By definition of the Yosida approximation,

$$
\phi^{\varepsilon}(x)=\frac{x-R^{\varepsilon}(x)}{\varepsilon},
$$

it is now easy to conclude that

$$
\phi^{\varepsilon}(x)= \begin{cases}0, & |x| \leq 1 \\ \frac{x-1}{\varepsilon} & x \in(1,1+\varepsilon] \\ \frac{x+1}{\varepsilon} & x \in[-1-\varepsilon, 1) \\ \frac{x}{1+\varepsilon} & |x|>1+\varepsilon\end{cases}
$$

In particular, for $\varepsilon \leq 1$ and $|x| \geq 1+\varepsilon$, we observe that

$$
\left|\phi^{\varepsilon}(x)\right| \geq \frac{|x|}{2} .
$$

\section{References}

1. Arnold, L., Chueshov, I.: Order-preserving random dynamical systems: equilibria, attractors, applications. Dyn. Stabil. Syst. 13, 265-280 (1998)

2. Barbu, V.: Nonlinear Differential Equations of Monotone Types in Banach Spaces. Springer, New York (2010)

3. Barbu, V.: Self-organized criticality of cellular automata model; absorbtion in finite-time of supercritical region into the critical one. Math. Methods Appl. Sci. 36, 1726-1733 (2013)

4. Barbu, V., Da Prato, G., Röckner, M.: Stochastic porous media equations and self-organized criticality. Commun. Math. Phys. 285, 901-923 (2009)

5. Barbu, V., Da Prato, G., Röckner, M.: Stochastic Porous Media Equations. Lecture Notes in Mathematics. Springer International Publishing (2016)

6. Barbu, V., Da Prato, G., Röckner, M.: Existence of strong solutions for stochastic porous media equation under general monotonicity conditions. Ann. Probab. 37, 428-452 (2009)

7. Barbu, V., Da Prato, G., Röckner, M.: Stochastic nonlinear diffusion equations with singular diffusivity. SIAM J. Math. Anal. 41, 1106-1120 (2009) 
8. Barbu, V., Da Prato, G., Röckner, M.: Finite time extinction of solutions to fast diffusion equations driven by linear multiplicative noise. J. Math. Anal. Appl. 389, 147-164 (2012)

9. Barbu, V., Röckner, M.: Stochastic variational inequalities and applications to the total variation flow perturbed by linear multiplicative noise. Arch. Ration. Mech. Anal. 209, 797-834 (2013)

10. Beyn, W.J., Gess, B., Lescot, P., Röckner, M.: The global random attractor for a class of Stochastic porous media equations. Commun. Par. Differ. Equ. 36, 446-469 (2010)

11. Brézis, H.: Opérateurs maximaux monotones et semi-groupes de contractions dans les espaces de Hilbert. Notas de Matemática. North-Holland Publishing Company, North-Holland Mathematics Studies (1973)

12. Butkovsky, O., Scheutzow, M.: Couplings via comparison principle and exponential ergodicity of SPDEs in the hypoelliptic setting. Commun. Math. Phys. 379, 1001-1034 (2020)

13. Chueshov, I., Scheutzow, M.: Invariance and monotonicity for stochastic delay differential equations. Discrete Contin. Dyn. Syst. B 18, 1533 (2013)

14. Cranston, M., Gess, B., Scheutzow, M.: Weak synchronization for isotropic flows. Discrete Contin. Dyn. Syst. Ser. B 21, 3003-3014 (2016)

15. Crauel, H., Debussche, A., Flandoli, F.: Random attractors. J. Dyn. Differ. Equ. 9, 307-341 (1997)

16. Crauel, H., Flandoli, F.: Attractors for random dynamical systems. Probab. Theory Relat. Fields 100, 365-393 (1994)

17. Da Prato, G., Röckner, M.: Weak solutions to stochastic porous media equations. J. Evol. Equ. 4, 249-271 (2004)

18. Da Prato, G., Röckner, M.: Invariant measures for a stochastic porous medium equation. In: Stochastic Analysis and Related Topics in Kyoto: In honour of Kiyosi Itô, pp. 13-29. Mathematical Society of Japan, Tokyo, Japan (2004)

19. Da Prato, G., Röckner, M., Rozovskii, B.L., Wang, F.Y.: Strong solutions of stochastic generalized porous media equations: existence, uniqueness, and ergodicity. Commun. Par. Differ. Equ. 31, 277-291 (2006)

20. Da Prato, G., Zabczyk, J.: Ergodicity for Infinite Dimensional Systems. Lecture Note Series, London Mathematical Society, Cambridge University Press, Cambridge (1996)

21. Dareiotis, K., Gess, B., Tsatsoulis, P.: Ergodicity for stochastic porous media equations with multiplicative noise. SIAM J. Math. Anal. 52, 4524-4564 (2020)

22. Flandoli, F., Gess, B., Scheutzow, M.: Synchronization by noise. Probab. Theory Relat. Fields 168, 511556 (2017)

23. Flandoli, F., Gess, B., Scheutzow, M.: Synchronization by noise for order-preserving random dynamical systems. Ann. Probab. 45, 1325-1350 (2017)

24. Gess, B.: Random attractors for degenerate stochastic partial differential equations. J. Dyn. Differ. Equ. 25, 121-157 (2013)

25. Gess, B.: Random attractors for singular stochastic evolution equations. J. Differ. Equ. 255, 524-559 (2013)

26. Gess, B.: Finite time extinction for stochastic sign fast diffusion and self-organized criticality. Commun. Math. Phys. 335, 309-344 (2015)

27. Gess, B., Liu, W., Röckner, M.: Random attractors for a class of stochastic partial differential equations driven by general additive noise. J. Differ. Equ. 251, 1225-1253 (2011)

28. Gess, B., Liu, W., Schenke, A.: Random attractors for locally monotone stochastic partial differential equations. arXiv e-prints arXiv:1908.03539 (2019)

29. Gess, B., Röckner, M.: Stochastic variational inequalities and regularity for degenerate stochastic partial differential equations. Trans. Am. Math. Soc. 369, 3017-3045 (2017)

30. Gess, B., Röckner, M.: Singular-degenerate multivalued stochastic fast diffusion equations. SIAM J. Math. Anal. 47, 4059-4090 (2015)

31. Gess, B., Tölle, J.M.: Multi-valued, singular stochastic evolution inclusions. Journal de Mathématiques Pures et Appliquées 101, 789-827 (2014)

32. Gess, B., Tölle, J.M.: Ergodicity and local limits for stochastic local and nonlocal $p$-laplace equations. SIAM J. Math. Anal. 48, 4094-4125 (2016)

33. Gilbarg, D., Trudinger, N.: Elliptic Partial Differential Equations of Second Order. Classics in Mathematics. Springer, Berlin (2001)

34. Hairer, M., Mattingly, J.C.: Ergodicity of the 2D Navier-Stokes equations with degenerate stochastic forcing. Ann. Math. 164, 993-1032 (2006)

35. Komorowski, T., Peszat, S., Szarek, T.: On ergodicity of some Markov processes. Ann. Probab. 38, 1401-1443 (2010)

36. Lasota, A., Szarek, T.: Lower bound technique in the theory of a Stochastic differential equation. J. Differ. Equ. 231, 513-533 (2006)

37. Liu, W.: Harnack inequality and applications for Stochastic evolution equations with monotone drifts. J. Evol. Equ. 9, 747-770 (2009) 
38. Liu, W., Röckner, M.: Stochastic Partial Differential Equations: An Introduction. Universitext, Springer International Publishing, Berlin (2015)

39. Liu, W., Tölle, J.M.: Existence and uniqueness of invariant measures for stochastic evolution equations with weakly dissipative drifts. Electron. Commun. Probab. 16, 447-457 (2011)

40. Neuß, M.: Well-posedness of svi solutions to singular-degenerate stochastic porous media equations arising in self-organised criticality. arXiv e-prints arXiv:2002.01220 (2020)

41. Prévot, C., Röckner, M.: A Concise Course on Stochastic Partial Differential Equations. Springer, Berlin (2007)

42. Revuz, D., Yor, M.: Continuous Martingales and Brownian Motion. Grundlehren der mathematischen Wissenschaften. Springer, Berlin (2004)

43. Rosati, T.C.: Synchronization for KPZ. arXiv e-prints arXiv:1907.06278 (2019)

44. Vazquez, J.: The Porous Medium Equation: Mathematical Theory. Oxford Mathematical Monographs, Clarendon Press, Oxford (2006)

45. Wang, F.Y.: Harnack inequality and applications for stochastic generalized porous media equations. Ann. Probab. 35, 1333-1350 (2007)

46. Wang, F.Y.: Asymptotic couplings by reflection and applications for nonlinear monotone SPDES. Nonlinear Anal. 117, 169-188 (2015)

47. Wang, F.Y.: Exponential convergence of non-linear monotone SPDEs. Discrete Contin. Dyn. Syst. 35, 5239-5253 (2015)

48. Watkins, N.W., Pruessner, G., Chapman, S.C., Crosby, N.B., Jensen, H.J.: 25 years of self-organized criticality: Concepts and controversies. Space Sci. Rev. 198, 3-44 (2016)

49. Ziemer, W.P.: Weakly Differentiable Functions: Sobolev Spaces and Functions of Bounded Variation. Springer, New York (1989)

Publisher's Note Springer Nature remains neutral with regard to jurisdictional claims in published maps and institutional affiliations. 